\title{
Constraints to Uranus' Great Collision III: The Origin of the Outer Satellites
}

\author{
Adrián Brunini and Mirta Gabriela Parisi \\ Observatorio Astronómico de La Plata, Paseo del Bosque s/n, 1900 La Plata, Argentina; and Comisión Nacional de Investigaciones Científicas y Técnicas \\ E-mail: gparisi@fcaglp.fcaglp.unlp.edu.ar
}

and

Gonzalo Tancredi

Facultad de Ciencias, Universidad de la República, Iguá 4225, 11400 Montevideo, Uruguay

Received July 17, 2000; revised April 22, 2002

The theory of satellite loss resulting from a giant impact on Uranus (Parisi and Brunini 1997, Planet. Space Sci. 45, 181-187) is revisited, in the light of the discovery of its five outer moons (Gladman et al. 1998, Nature 392, 897-899; Gladman et al. 2000, Icarus 147, 320-324; erratum 148, 320). Physical conditions and dynamical constraints in the great collision scenario and restrictions in the possible mechanisms for the origin of the outer uranian satellites are obtained from the knowledge of their actual orbital properties. We conclude that the existence of these moons implies that their origin must be connected to a breakup process. Other scenarios for their origin cast serious doubts on the occurrence of a giant collision at the end of Uranus' formation process to account for its large spin axis obliquity. (c) 2002 Elsevier Science (USA)

Key Words: Uranus; satellites of uranus; orbits; origin, Solar System.

\section{INTRODUCTION}

The giant planets of the Solar System have remarkable resemblances, suggesting a similar formation scenario. It is usually accepted that the cores of the giant planets were formed by the accretion of planetesimals (Bodenheimer and Pollack 1986, Pollack et al. 1996). The present eccentricity and inclination of their orbits are mainly determined by this process of accumulation, i.e., by the momentum acquired by the planets due to encounters and collisions with the distribution of planetesimals during the accretion phase (Ziglina 1976, Ziglina and Safronov 1976, Harris and Ward 1982, Parisi and Brunini 1999). In this process of accumulation, planets also acquire spin angular momentum from the relative motion of planetesimals at impact. Dones and Tremaine (1993), Lissauer and Kary (1991), and Lissauer et al. (1997) computed the rotation rate of a planet which accretes from a disk of small, solid planetesimals. This seems to be the origin of the systematic component of planetary rotation. However, the rotation axes of the planets were tilted from the direction perpendicular to the orbital plane, as a consequence of stochastic off-center impacts onto the planets during the accretion phase (Safronov 1969, Lissauer and Safronov 1991, Brunini 1993, Dones and Tremaine 1993). Nevertheless, Uranus is the only giant planet with a large spin axis inclination $\left(98^{\circ}\right)$. This large obliquity is usually attributed to a great tangential collision with another protoplanet when Uranus was at the end of its formation process (Korycansky et al. 1990, Brunini 1995a, Parisi and Brunini 1996a, Parisi and Brunini 1997 (hereafter $\mathrm{PB})$ ) or perhaps significantly later in the evolution of the planet (Slattery et al. 1992). It should be mentioned that other scenarios to account for the inclination of the spin axis of Uranus have been proposed. Kubo-Oka and Nakazawa (1995) investigated the tidal evolution of satellite orbits and examined the possibility that the orbital decay of a retrograde satellite leads to the large obliquity of Uranus, but the large mass required for the hypothetical satellite makes this possibility very implausible. An asymmetric infall or torque from nearby mass concentrations during the collapse of the molecular cloud core that led to the formation of the Solar System could twist the total angular momentum vector of the planetary system. This twist could generate the obliquities of the outer planets (Tremaine 1991), but this model has the disadvantage that the outer planets must form before the infall is complete. Harris and Ward (1982) found that if a slow change in either the axial or the orbital precession frequencies occurred since the formation of the planets, then the resulting resonances might have altered the direction of the spin axes of the planets. The chaotic obliquity of the planets was carefully investigated by Laskar and Robulet (1993); they showed that the obliquities of the giant planets are essentially stable along their entire orbital evolution. Thus, it is not possible to explain the very large obliquity of Uranus by a chaotic behavior of its rotation axis. At the end of the runaway growth of planetesimals, gravitational perturbations by more than one 
protoplanet can excite sufficient random velocities to substantially slow the rate of growth. Once the protoplanets have perturbed one another into crossing orbits, their subsequent orbital evolution is governed by close gravitational encounters and violent, highly inelastic collisions (Lissauer 1993, Levison et al. 1998, Brunini and Fernández 1999). These encounters and impacts alter the planetary orbits (Parisi and Brunini 1996b, 1999) and the latter mainly change the direction of the spin axes of the planets (Safronov 1969, Lissauer and Safronov 1991). Within this scenario, we argue that the large obliquity of Uranus may be due to a great collision. Stevenson et al. (1986) pointed out that such an impact would excite the orbital eccentricity of Uranus. However, Parisi and Brunini (1996b, 1999) showed that an impactor mass less than 1.6-1.9m $m_{\oplus}$ would excite an orbital eccentricity for Uranus lower than its present value.

The rich satellite system of the giant planets have common features, since their existence is probably due to similar formation and evolutionary processes. Close to each planet there are the "regular satellites" orbiting close to the equatorial plane of the planet in almost circular orbits. Far away from each giant planet the "irregular satellites" orbit in elongated and inclined orbits. The gap between regular and irregular satellites is connected to different formation processes. It is generally accepted that, unlike the regular satellites, the irregular satellites did not form in situ. The regular satellites accreted within circumplanetary disks, but the irregular satellites are thought to have been captured from orbits around the Sun. Hitherto, the only departure from this broad classification was the satellite system around Uranus, in which no irregular satellites were known.

Stevenson et al. (1986) argue that the existence of the satelliteforming disks is a general consequence of Solar System evolution for Jupiter and Saturn. But for the origin of the regular satellites of Uranus, they state that Uranus' protosatellite disk may be a special case arising from the giant impact, which has given Uranus its large obliquity. Slattery et al. (1992) have modeled the effects of a giant impact on Uranus. The smallest impactor mass in these simulations was $1 m_{\oplus}$. Slattery et al. (1992) found that sufficient material to account for the uranian regular satellite system can be placed into orbit by such an impact. It should be mentioned that Korycansky et al. (1990) state that, because of the sharpness of the transition between envelope retention and expulsion, forming an accretion disk as precursor of the regular uranian satellites by a blowout resulting from the collision might be difficult. It is not probable that the present regular satellites of Uranus existed before collision, because there is no known efficient mechanism able to bring their orbital inclination from $0^{\circ}$ to its present value of $98^{\circ}$ (Greenberg 1974).

It is possible for an object in heliocentric motion to be temporarily trapped as a planetary satellite. In terms of the classical three-body problem this type of capture can occur when the object passes through the interior Lagrangian point, $L_{2}$, with a very low relative velocity. But, without any other mechanism, such a capture is not permanent and the object will eventually return to a solar orbit. Numerical studies confirm this, indicat- ing that typically the capture intervals last from several to several hundred orbital periods about the planet (Byl and Ovenden 1975, Heppenheimer and Porco 1977). However, a stable satellite is able to survive for more than 1000 orbits around the planet without escaping from the planet's sphere of influence (Huang and Innanen 1983, Brunini 1996). To turn a temporary capture into a permanent one, it is necessary to dissipate orbital energy. Capture theories for the irregular satellites fall mainly into two categories. In one, the satellites are captured by dissipative forces such as gas drag or tidal friction, but because of the large distance of the irregular satellites from their parent planets, tidal friction seems to be implausible (Pollack et al. 1979, Goldreich et al. 1989, Pollack et al. 1991, Saha and Tremaine 1993, McKinnon and Leith 1995). Such forces could fracture a single body to form a cluster of satellites, as in the case of the two groups of outer satellites of Jupiter. The second category of capture theory involves a collision between a planetesimal and a satellite (Colombo and Franklin 1971). The collision shatters both bodies, creating fragments that are then captured into planetocentric orbits. Large temporal variations in the brightnesses of irregular satellites are expected from rotating bodies of highly elongated shapes consistent with a collision fragment origin. The lightcurve amplitudes of the main belt asteroids are consistent with this scenario (Catullo et al. 1984). Pollack et al. (1979) attributed the relatively small brightness variations of the jovian outer satellites to smoothing by ablation during gas drag capture. However, current observations of Luu (1991) point out that the Pollack et al. (1979) prediction is not unique. Luu (1991) showed that the brightness variations of the observed jovian satellites are consistent with those of main belt asteroids, which are collision fragments with randomized spin vectors. The photometric properties of the outer jovian satellites (Tholen and Zellner 1984) resemble main belt asteroids of type C (Degewij et al. 1980). Other mechanisms to turn a temporary capture into a permanent one have been studied. Such a transition could occur if the mass of the planet increased and/or the mass of the Sun decreased during the temporary capture phase (Heppenheimer 1975, Byl and Ovenden 1975). The disadvantage of this mechanism is the sudden mass changes needed during the temporary capture. Brunini (1995b) studied the enhancement of the Hill sphere of action not only due to the mass acquired by the planet but also by the variation of the planetSun distance as a consequence of the scattering of planetesimals by the outer Solar System. Tsui (1999) suggested a scattering mechanism by a preexisting planetary satellite to account for permanent capture of guest bodies. But no single explanation has been able to account for the dissimilar sizes and configurations of the irregular satellites (Luu 1997).

Until very recently, no irregular satellites of Uranus were known. We showed in PB that the great collision could explain the lack of outer satellites of the planet. If the great collision hypothesis is accepted for the origin of the large spin axis inclination of Uranus, it might have had important consequences on the uranian satellite system if it were present prior to the event. The 
impulse imparted to Uranus at collision would produce a shift in the planetary orbital velocity. The preexisting satellites would suffer the same orbital change with respect to Uranus' center of mass. The velocity change and the mass of the impactor may be estimated from simple considerations about the dynamics of the collision (PB). The outermost satellites would become unbound; inner satellites could suffer ejection or change into more or less bound orbits depending on the geometry of the impact and the orbit and velocity of the satellite at the moment of impact. The main result of $\mathrm{PB}$ was that any particle orbiting Uranus beyond $\sim 70-90$ planetary radii $\left(R_{\mathrm{U}}\right)$ was probably swept out from the system. Then, we concluded that the existence of outer satellites of Uranus was hardly expected and that a further intensive search for very faint objects orbiting Uranus beyond 90 planetary radii would provide a constraint to the great collision scenario.

Later, on 31 October 1997, two outer satellites of Uranus, S/1997 U1 (Caliban) and S/1997 U2 (Sycorax) were discovered by Gladman et al. (1997, 1998). The first orbital computations were carried out by Marsden and Williams (1997). The actual orbital parameters of S/1997 U1 and S/1997 U2, computed by Marsden et al. (1998a,b), are shown in Table I. In 1999, Kavelaars and collaborators (1999) reported the discovery of two new outer satellites of Uranus, S/1999 U1 (Setebos) and S/1999 U2 (Stephano). Gladman et al. (1999) reported new observations of both satellites together with observations of a third satellite, S/1999 U3 (Prospero). The discovery of these three moons has been published in Gladman et al. (2000). Their orbital elements are included in Table I (Marsden 2000a,b,c).

The recently discovered Centaur and Kuiper belt objects show a wide range of reddish colors (Luu and Jewitt 1996), encompassing those of S/1997 U1 and S/1997 U2 (Gladman et al. 1998), and perhaps resulting from the bombardment of organicrich icy surfaces by energetic particles (Wilson 1997). An alternative model, assuming red initial surfaces due to an organic-rich composition to account for the wide range of reddish colors, is developed by Gil Hutton (2001). The fact that S/1997 $\mathrm{U}_{1}$ and $S / 1997 U_{2}$ are red suggests that the outer uranian satellites are escapees from the Kuiper belt (Duncan and Levison 1997, Morbidelli 1997).

The discovery of the outer uranian satellites provides important clues in the great collision scenario. Physical conditions and dynamical constraints at the last stage of Uranus' formation process are settled from the knowledge of the physical and orbital properties of the outer uranian moons. The possible scenarios for the origin of these satellites are then established.

The stochastic processes and their consequences on the physical and orbital properties of the planets have been long known. However, the study of how these processes may affect preexisting satellite systems is a new concept introduced by Brunini (1995a) and developed only in PB, in Parisi's Ph.D. thesis studies, and in the present work. In this spirit, the richness of the irregular satellite systems (i.e., Gladman et al. 2001, Marsden 2000d,e, Green 2001) yields new insights into the stochastic events and may set physical conditions and strong constraints in the scenario of the formation of the giant planets.

\section{SUMMARY OF PARISI AND BRUNINI (1997)}

We assumed that the spin axis inclination of Uranus was caused by an inelastic, off-center collision onto the planet when Uranus was at the end of its accretion phase. In this stage of Uranus' formation, the planet's envelope extends to $\sim 6.5 \times$ $10^{6} \mathrm{~km}$ (Korycansky et al. 1990), whereas the core containing most of the mass has a radius of only $\sim 1.8 \times 10^{4} \mathrm{~km}$. In this situation, a collision with the core itself is necessary to impart the required mass and angular momentum (Korycansky et al. 1990). Since the impact parameter of the collision $b$ is an unknown quantity, we took its most probable value: $b=(2 / 3) R_{\mathrm{c}}$, where $R_{\mathrm{c}}$ is the core radius of Uranus at the moment of collision.

Equation (8) of PB gives a relation between the impactor's mass $m_{\mathrm{i}}$ and its incident speed $v_{\mathrm{i}}$ from angular momentum conservation,

$$
\begin{aligned}
v_{\mathrm{i}}= & \frac{3\left(m_{\mathrm{U}}+m_{\mathrm{i}}\right)}{5 m_{\mathrm{i}} R_{\mathrm{c}}} R_{\mathrm{U}}^{2}\left[\Omega^{2}+\frac{\Omega_{0}^{2}}{\left(1+\frac{m_{\mathrm{i}}}{m_{\mathrm{U}}}\right)^{2}\left(1+\frac{m_{\mathrm{i}}}{3 m_{\mathrm{U}}}\right)^{4}}\right. \\
& \left.-\frac{2 \Omega \Omega_{0} \cos \alpha}{\left(1+\frac{m_{\mathrm{i}}}{m_{\mathrm{U}}}\right)\left(1+\frac{m_{\mathrm{i}}}{3 m_{\mathrm{U}}}\right)^{2}}\right]^{1 / 2},
\end{aligned}
$$

where $\left(m_{\mathrm{U}}+m_{\mathrm{i}}\right)$ is the present mass of Uranus and $R_{\mathrm{U}}$ is its current radius. Also, $\vec{\Omega}$ is Uranus' present angular velocity of

TABLE I

\begin{tabular}{|c|c|c|c|c|c|}
\hline IAU designation & S/1997 U1 & S/1997 U2 & S/1999 U1 & S/1999 U2 & S/1999 U3 \\
\hline \multirow[t]{2}{*}{ Provisional name } & U XVI & U XVII & U XIX & UXX & U XVIII \\
\hline & Caliban & Sycorax & Setebos & Stephano & Prospero \\
\hline$r_{\mathrm{s}}(\mathrm{km})(p=0.07)$ & 30 & 60 & 15 & 10 & 15 \\
\hline$e$ & 0.082 & 0.509 & 0.528 & 0.146 & 0.323 \\
\hline$a\left(R_{\mathrm{U}}\right)$ & 283 & 482 & 703 & 312 & 658 \\
\hline Inclination $\left(^{\circ}\right)$ & 139.7 & 152.7 & 147.7 & 141.5 & 146.2 \\
\hline Period (days) & 579 & 1289 & 2271 & 674 & 2057 \\
\hline Ref. orbit & IAUC 6870 & IAUC 6869 & IAUC 7450 & IAUC 7473 & IAUC 7447 \\
\hline
\end{tabular}

The Outer Satellites of Uranus 
TABLE II

Range of Impactor Masses Required to Tilt Uranus' Spin Axis

\begin{tabular}{rrcc}
\hline$T_{0}(\mathrm{~h})$ & $\alpha\left(^{\circ}\right)$ & $m_{\mathrm{i}, \max }\left(m_{\oplus}\right)$ & $m_{\mathrm{i}, \min }\left(m_{\oplus}\right)$ \\
\hline 20 & 70 & 1.6 & 1 \\
20 & 130 & 2.4 & 1.6 \\
9 & 70 & 2.2 & 1.5 \\
9 & 130 & 3.2 & 2.3 \\
\hline
\end{tabular}

rotation, while $\vec{\Omega}_{0}$ is the spin angular velocity which it would have today if the great collision had never occurred. Finally, $\alpha$ is the angle between $\vec{\Omega}$ and $\vec{\Omega}_{0}$.

It should be noted that $\Omega_{0}$ and $\alpha$ are unknown quantities. We constrained these parameters assuming that the original spin obliquity and period of Uranus should be similar to the other giant planets by taking two values for $\alpha$ of $70^{\circ}$ and $130^{\circ}$ and for $T_{0}\left(=2 \pi / \Omega_{0}\right)$ of 9 and $20 \mathrm{~h}$.

To obtain constraints on the impactor incident speed, we considered that the impactor had a hyperbolic orbit with respect to Uranus before the impact. The maximum allowed incident impactor velocity was taken as that of an initially heliocentric parabolic object lying in the same orbital plane as the protoUranus and moving in a direction opposite to Uranus' motion, including the acceleration caused by the planet. This maximum allowed value is $v_{\mathrm{i}} \sim 31.54 \mathrm{~km} \mathrm{~s}^{-1}$. The minimum allowed impactor incident velocity corresponds to a null relative velocity at infinity. Due to the acceleration caused by Uranus, the minimum value of $v_{\mathrm{i}}$ is $\sim 20 \mathrm{~km} \mathrm{~s}^{-1}$. Using these constraints, the maximum and minimum impactor masses required to tilt Uranus' spin axis are obtained from Figs. 1 and 2 of PB. These results are shown in Table II.

Equation (15) of PB gives the minimum orbital radius $r_{\mathrm{m}}$ from which any satellite in an originally circular orbit would be unbound by the impulse imparted in the collision,

$$
r_{\mathrm{m}}=\frac{G m_{\mathrm{U}}}{(\Delta V)^{2}}\left[\frac{1+2 \frac{m_{\mathrm{i}}}{m_{\mathrm{U}}}}{\cos \psi+\sqrt{\cos \psi^{2}+1+2 \frac{m_{\mathrm{i}}}{m_{\mathrm{U}}}}}\right]^{2},
$$

where $G$ is the gravitational constant and $\Delta V$ is the orbital velocity change suffered by Uranus, $\psi$ being the angle between $\Delta \vec{V}$ and the circular orbital velocity of the satellite. Through momentum conservation considerations at collision, the value of $\Delta V$ may be obtained from Eq. (10) of PB:

$$
\left(m_{\mathrm{i}}+m_{\mathrm{U}}\right) \Delta \vec{V}=m_{\mathrm{i}} \vec{v}_{\mathrm{i}}
$$

As $\psi$ is unknown, we calculated in PB the average of $r_{\mathrm{m}}$ over all the space to obtain the most probable value of $r_{\mathrm{m}}\left(\left\langle r_{\mathrm{m}}\right\rangle\right)$. The upper constraint of $\left\langle r_{\mathrm{m}}\right\rangle$, shown in Fig. 4 of PB, was $\sim 70-90$ (in units of the present radius of the planet, $R_{\mathrm{U}}=25,400 \mathrm{~km}$ ), which corresponds to $T_{0}=20 \mathrm{~h}$ and $\alpha=70^{\circ}$.
The case of initial elliptical orbits for the satellites reaching the escape velocity at impact was also considered. We calculated the most probable value of the minimum orbital semiaxis, $\left\langle a_{\mathrm{m}}\right\rangle$, for different values of the orbital eccentricity of the satellites. We found that $a_{\mathrm{m}}$ is a function not only of $\psi$ but also of the eccentricity and the position of the satellite on its orbit. Then, we averaged over all the possible incident directions of the impactor and also over time along the satellite orbit, obtaining $\left\langle a_{\mathrm{m}}\right\rangle$. However, the resulting value of $\left\langle a_{\mathrm{m}}\right\rangle$ was independent of the orbital eccentricity of the satellite, $\left\langle a_{\mathrm{m}}\right\rangle=\left\langle r_{\mathrm{m}}\right\rangle$. The most probable value of the minimum orbital semiaxis from which any satellite is unbound by the collision is $\sim 70-90 R_{\mathrm{U}}$ for any initial eccentricity.

However, the discovery of the outer uranian moons leads us to a rigorous analysis of the behavior of the satellites' orbits, instead of a probabilistic study. When no averages are taken, the destiny of the satellites' orbits depends on the orbital eccentricity, the position of the satellite in its orbit at the moment of collision, and the direction of the impactor incident velocity. The detailed study of this problem is the topic of the following sections.

\section{INITIAL CIRCULAR ORBITS}

New dynamical constraints to Uranus' great collision hypothesis are settled assuming circular orbits for the satellite orbits before the impact. Upper and lower bounds in $r_{\mathrm{m}}$ (as a function of the impactor mass $m_{\mathrm{i}}$ ) are obtained by setting $\psi=180^{\circ}$ and $\psi=0^{\circ}$, respectively, in Eq. (2). These results are displayed in Fig. 1 for $T_{0}=20 \mathrm{~h}$, where the most probable value of $r_{\mathrm{m}}\left(\left\langle r_{m}\right\rangle\right)$

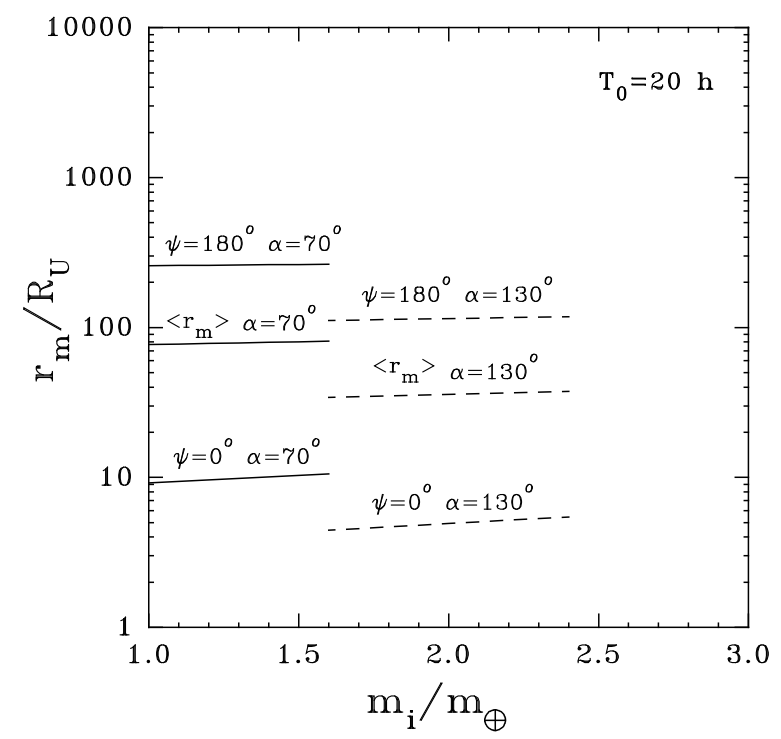

FIG. 1. Upper bound, most probable value, and lower bound (from top to bottom) of the minimum orbital semiaxis $r_{\mathrm{m}}$ (in units of $R_{\mathrm{U}}=25,400 \mathrm{~km}$ ), as a function of the impactor mass $m_{\mathrm{i}}$ (in the range given in Table II) for $T_{0}=20 \mathrm{~h}$. Solid lines correspond to the case of an inclination $\alpha=70^{\circ}$, while dashed lines depict the results corresponding to $\alpha=130^{\circ}$. Initial circular orbits for the satellites which are unbound due to the impulse imparted in the collision are considered. 


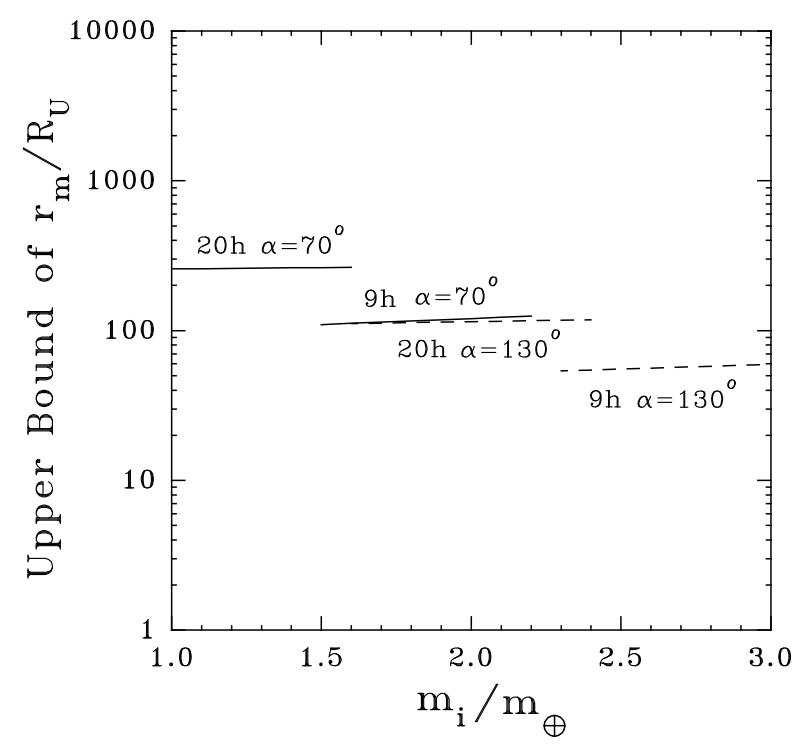

FIG. 2. Upper bounds of $r_{\mathrm{m}}$ (in units of $R_{\mathrm{U}}=25,400 \mathrm{~km}$ ) for $T_{0}=9$ and $20 \mathrm{~h}$ as a function of the impactor mass $m_{\mathrm{i}}$ (in the range given in Table II). Solid lines correspond to $\alpha=70^{\circ}$, while dashed lines depict the results for $\alpha=130^{\circ}$. Initial circular orbits for the satellites which are unbound due to the impulse imparted in the collision are considered.

displayed in Fig. 4 of PB is also shown in Fig. 1. For intermediate values of $\psi, r_{\mathrm{m}}$ falls between the bounds.

In Fig. 2, the upper bounds in $r_{\mathrm{m}}$ for $T_{0}=9$ and $20 \mathrm{~h}$ are shown. The highest curve corresponds to $T_{0}=20 \mathrm{~h}$ and $\alpha=70^{\circ}$, where any satellite in an initially circular orbit with semiaxis equal to or greater than $r_{\mathrm{m}} \sim 250-270 R_{\mathrm{U}}$ would be unbound in the collision for every incident direction of the impactor. However, the actual semiaxes of the outer satellites of Uranus are greater than $270 R_{\mathrm{U}}$ (see Table I). We have to find the parameters able to keep satellites orbiting at the observed distances of the outer uranian moons. If $\alpha=70^{\circ}$ and $T_{0}=30 \mathrm{~h}$, the upper bound in $r_{\mathrm{m}}$ is $\sim 300 R_{\mathrm{U}}$. For each $\alpha<90^{\circ}$, it is possible to find the value of $T_{0}=T_{0 \mathrm{~m}}$ that maximizes Eq. (2). If $\alpha=70^{\circ}$, $T_{0 \mathrm{~m}}=45 \mathrm{~h}$. In this case any satellite in a circular orbit with semiaxis greater than $310 R_{\mathrm{U}}$ is unbound for any initial period (even if the initial period is infinite). But the satellite S/1999 U3 has a larger pericentric distance $\left(\sim 380 R_{\mathrm{U}}\right)$. It is necessary to reduce $\alpha$ to keep any satellite at that radius. This occurs when $\alpha=55^{\circ}$, where $T_{0 \mathrm{~m}}=27 \mathrm{~h}$ and any circular orbit from $\sim 400 R_{\mathrm{U}}$ is unbound for any initial period and any incident direction of the impactor. Such a small obliquity due to the impact could argue against the great collision hypothesis as a mechanism to explain the large spin obliquity of Uranus, if we assume that S/1999 U3 existed before the collision at its present distance.

Because satellites in near-circular orbits at these radii inside the range of expected parameters would have been then unbound, let us consider a scenario in which the collision increases the semimajor axis of an originally more tightly bound satellite. Following PB, we relate the initial circular orbit's radius $a_{\mathrm{c}}$ to its final (and current) semimajor axis $a$ via

$$
a_{\mathrm{c}}=\frac{G m_{\mathrm{U}}}{(\Delta V)^{2}}\left[\frac{1+2 \frac{m_{\mathrm{i}}}{m_{\mathrm{U}}}}{\cos \psi+\sqrt{\cos \psi^{2}+\left(1+2 \frac{m_{\mathrm{i}}}{m_{\mathrm{U}}}\right)\left(1+\frac{G\left(m_{\mathrm{U}}+m_{\mathrm{i}}\right)}{a(\Delta V)^{2}}\right)}}\right]^{2} .
$$

Upper and lower bounds in $a_{\mathrm{c}}$ are obtained by setting $\psi=$ $180^{\circ}$ and $\psi=0^{\circ}$, respectively, in Eq. (4). These bounds, as well as the most probable value of $a_{\mathrm{c}}\left(\left\langle a_{\mathrm{c}}\right\rangle\right)$ for S/1999 U3 and $T_{0}=20 \mathrm{~h}$ are displayed in Fig. 3. For $T_{0}=9 \mathrm{~h}$, the curves become lower than these ones. The minimum eccentricity acquired by any orbit after collision is $e_{\mathrm{m}}=1-a_{\mathrm{c}} / a$, where the upper bound of $a_{\mathrm{c}}$ must be used. In Fig. 4, we display the results of $e_{\mathrm{m}}$ for $T_{0}=9$ and $20 \mathrm{~h}$, and for $\alpha=70^{\circ}$ and $130^{\circ}$ for the transfer to S/1999 U3 orbit. The lowest curve of the minimum eccentricity corresponds to the upper curve of Fig. 3, where any satellite in circular orbit with initial semiaxis less than or equal to $\sim 230 R_{\mathrm{U}}$ might reach the same orbital semiaxis as S/1999 U3, exciting an orbital eccentricity higher than $e_{\mathrm{m}} \sim 0.64$.

The orbital evolution of the uranian satellites over a period of $3 \times 10^{5}$ years was computed by means of the numerical integration of the equations of the elliptical restricted three-body problem, formed by the Sun, Uranus, and the satellite. The variations of the semiaxes proved negligible for all the satellites. The minimum, mean, and maximum eccentricities along this period

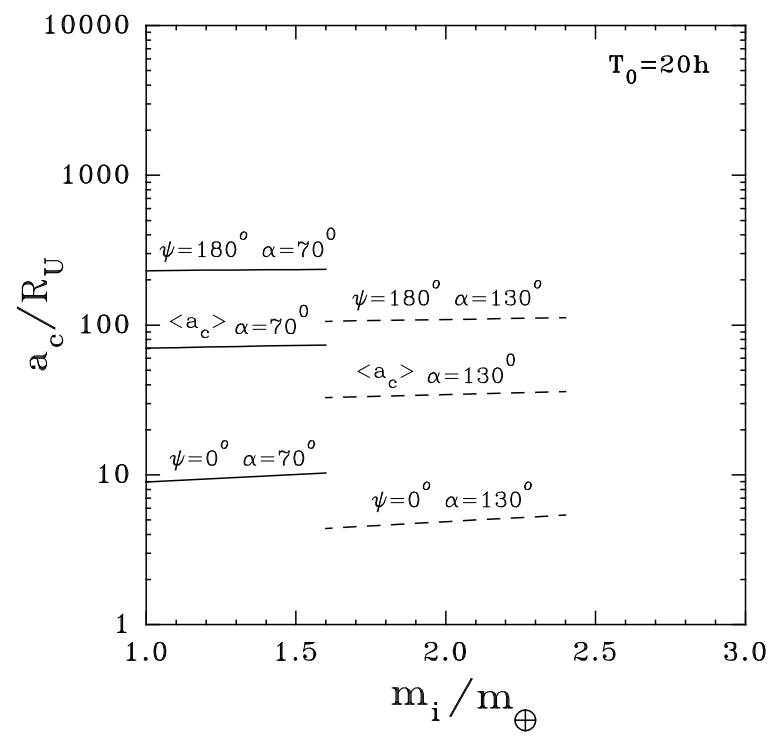

FIG. 3. Upper bound, most probable value, and lower bound (from top to bottom) of the semiaxis $a_{\mathrm{c}}$ (initial circular orbit in units of $R_{\mathrm{U}}=25,400 \mathrm{~km}$ ) of an object which is transferred to S/1999 U3 orbit. These results are shown as a function of the impactor mass $m_{\mathrm{i}}$ (in the range given in Table II) for $T_{0}=20 \mathrm{~h}$. Solid lines correspond to the case of an inclination $\alpha=70^{\circ}$, while dashed lines correspond to $\alpha=130^{\circ}$. 


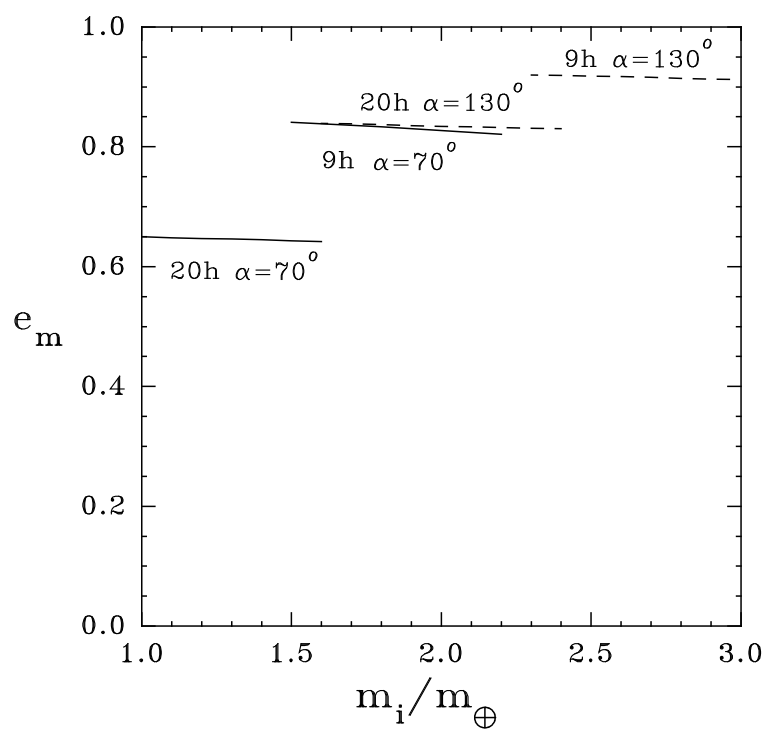

FIG. 4. The minimum eccentricity $e_{\mathrm{m}}$ acquired in the transfer from an initial circular orbit to the semiaxis of S/1999 U3 shown for $T_{0}=9$ and $20 \mathrm{~h}$. Solid lines correspond to the case of an inclination $\alpha=70^{\circ}$, while dashed lines correspond to $\alpha=130^{\circ}$. The lowest curve of the minimum eccentricity corresponds to the highest curve of the upper bound of $a_{\mathrm{c}}$, which is for $T_{0}=20 \mathrm{~h}$ and $\alpha=70^{\circ}$ (see Fig. 3).

for the five satellites are shown in Table III. These results are, generally speaking, in good agreement with those obtained by Jacobson $(1999,2001)$ with a much more sophisticated model. The maximum eccentricity of S/1999 U3 $\left(e_{\max }=0.54\right)$ is lower than $e_{\mathrm{m}} \sim 0.64$ and thus the transfer is not possible. The transfers for the other outer satellites are possible, since the resulting minimum orbital eccentricities are lower than their current maximum values. However, even when an initial period $T_{0 \mathrm{~m}}$ is considered (for $\alpha=70^{\circ}$ ), the transfer to S/1999 U3's orbit excites an orbital eccentricity higher than $e_{\max }$. We could invoke gas drag to damp the orbital eccentricity after collision. However, as we will see in Section 5, the effect of gas drag is negligible $\left(\right.$ de $\left./ e \sim 10^{-3}\right)$.

Under the simplified scenario studied in this section, the satellite S/1999 U3 would refute the hypothesis of its existence before the impact. In the next section, we will consider a more realistic model, where original elliptical orbits of the uranian satellites are introduced.

\section{TABLE III}

Variation of the Eccentricity due to Solar Perturbations

\begin{tabular}{lccc}
\hline Satellite & $e_{\min }$ & $e_{\text {mean }}$ & $e_{\max }$ \\
\hline S/1997 U1 & 0.07 & 0.19 & 0.33 \\
S/1997 U2 & 0.46 & 0.53 & 0.62 \\
S/1999 U1 & 0.46 & 0.57 & 0.70 \\
S/1999 U2 & 0.13 & 0.24 & 0.35 \\
S/1999 U3 & 0.27 & 0.40 & 0.54 \\
\hline
\end{tabular}

\section{INITIAL ELLIPTICAL ORBITS}

When initial elliptical orbits are considered, the orbits after collision depend on the orbital eccentricity before the impact, the position of the satellite on its orbit at impact, and the incident direction of the impactor. We generalize the equations of PB, allowing any initial orbital eccentricity for the satellites, which are assumed to be at any point along their orbits at the moment of collision.

The square of the initial orbital velocity $v_{1}$ of a hypothetical satellite of negligible mass is

$$
v_{1}^{2}=G m_{\mathrm{U}}\left(\frac{2}{r}-\frac{1}{a_{1}}\right),
$$

where $r$ is the position of the satellite on its orbit at the moment of collision and $a_{1}$ is its orbital semiaxis before the impact. After the impact, the satellite is transferred to another orbit with semiaxis $a_{2}$, acquiring the velocity $v_{2}$, where

$$
v_{2}^{2}=G\left(m_{\mathrm{U}}+m_{\mathrm{i}}\right)\left(\frac{2}{r}-\frac{1}{a_{2}}\right) .
$$

Let $\psi$ be the angle between the orbital velocity of the satellite before the impact $\left(\vec{v}_{1}\right)$ and the velocity change imparted to the system $(\Delta \vec{V})$. The following relation is then fulfilled:

$$
v_{2}^{2}=v_{1}^{2}+(\Delta V)^{2}+2 v_{1} \Delta V \cos \psi .
$$

We set $v_{1}^{2}=A v_{\mathrm{e}}^{2}$ and $v_{2}^{2}=B\left(1+m_{\mathrm{i}} / m_{\mathrm{U}}\right) v_{\mathrm{e}}^{2}$, where $A$ and $B$ are arbitrary coefficients $(0<A \leq 1, B>0)$ and $v_{\mathrm{e}}$ is the escape velocity at $r$ before the impact. Note that when $A=1 / 2$, the initial orbit is circular. Then, the semiaxes of the orbits before and after collision satisfy the following simple relations:

$$
a_{1}=\frac{r}{2(1-A)}, \quad a_{2}=\frac{r}{2(1-B)} .
$$

If $A<B$ then $a_{1}<a_{2}$. In the special case of $B=1$, the satellite is unbound from the system. If $A>B$ then $a_{1}>a_{2}$, and the satellite is transferred to an interior orbit. When $A=B$, the orbital semiaxis remains unchanged $\left(a_{1}=a_{2}\right)$.

From Eqs. (5), (6), and (7), we arrive at a quadratic equation in $v_{\mathrm{e}}$ :

$$
v_{\mathrm{e}}=\Delta V \frac{\sqrt{A} \cos \psi \pm \sqrt{\left(B^{\prime}-A\right)+A \cos ^{2} \psi}}{B^{\prime}-A},
$$

where $B^{\prime}=B\left(1+m_{\mathrm{i}} / m_{\mathrm{U}}\right)$. Through the relation $r=2 G m_{\mathrm{U}} /$ $v_{\mathrm{e}}^{2}$, we may obtain an expression for $r$ :

$$
r=\frac{2 G m_{\mathrm{U}}}{(\Delta V)^{2}}\left[\frac{B^{\prime}-A}{\sqrt{A} \cos \psi \pm \sqrt{\left(B^{\prime}-A\right)+A \cos ^{2} \psi}}\right]^{2} .
$$

When $A=1 / 2$ and $B=1$ we arrive at Eq. (2). 
As $v_{\mathrm{e}}$ must be positive, we must consider two cases:

1. $A<B^{\prime}\left(v_{1}^{2}<v_{2}^{2}\right)$. These transfers lead to a less bound orbit. In this case the argument in the square root of Eq. (9) is always positive and the square root is greater than the first term. Therefore, only the positive sign is permitted. All values of $\psi$ are possible. Upper and lower bounds in $r$ as a function of $A$, $B, m_{\mathrm{i}}$, and $\Delta V$ are obtained setting $\psi=180^{\circ}\left(\vec{v}_{2}\right.$ has the same direction but opposite sense as $\left.\vec{v}_{1}\right)$ and $\psi=0^{\circ}\left(\vec{v}_{2}\right.$ has the same direction and sense as $\vec{v}_{1}$ ), respectively. For intermediate values of $\psi$, the results fall between both bounds. The upper and lower bounds of $r$ correspond to the lower and upper bounds of $v_{\mathrm{e}}, v_{1}$, and $v_{2}$, respectively.

2. $A>B^{\prime}\left(v_{1}^{2}>v_{2}^{2}\right)$. This condition means that the instantaneous orbital velocity is reduced. These transfers lead to a more bound orbit. In this case both signs of the square root in Eq. (9) are permitted, but a forbidden window in $\psi$ appears, because, to reduce the instantaneous satellite velocity, the impulse must be in the direction opposite to that of the satellite motion. Then, for a given $A$ and $B$, the incident directions of the impactor inside the window $\cos ^{2} \psi \geq\left(1-B^{\prime} / A\right)$ with $\cos \psi<0$ are only possible. Upper and lower bounds in $r$ as a function of $A, B, m_{\mathrm{i}}$, and $\Delta V$ are settled for the same value of $\psi=180^{\circ}$ but taking the positive and negative signs of the square root in the denominator, respectively. As in case 1, the upper bound for $r$ is found when $\vec{v}_{2}$ has the same direction as $\vec{v}_{1}$ and opposite sense, while for the lower bound $\vec{v}_{2}$ has the same direction and sense as $\vec{v}_{1}$.

For given $A, m_{\mathrm{i}}, \Delta V$, and $\cos \psi<0$, there are two solutions for $r$ (which correspond to both signs of the square root) as a function of $B$, which are interior to the upper and lower bounds. Both solutions approach each other as $B$ decreases, until they meet where $B^{\prime}=A\left(1-\cos ^{2} \psi\right)$. Then, the transfers to a more bound orbit have values of $B^{\prime} \geq A\left(1-\cos ^{2} \psi\right)$, which means that $v_{2}^{2} \geq v_{1}^{2}\left(1-\cos ^{2} \psi\right)$. A lower $B^{\prime}\left(\right.$ smaller $\left.v_{2}\right)$ would require a larger impulse in the direction opposite to that of the satellite motion (a larger $\cos ^{2} \psi$ ) and then, the permitted window would be smaller. If $\psi=180^{\circ}$, all values of $B^{\prime}<A$ are possible. If $\psi=90^{\circ}$ or $270^{\circ}$, the permitted window would imply that $B^{\prime}>A$, returning to case 1 .

The transition between the cases 1 and 2 occurs when $A=B^{\prime}$. Then, $v_{1}^{2}=v_{2}^{2}$. At this critical radius $r_{\text {crit }}$, Eq. (10) cannot be applied and the following expression is obtained from Eqs. (5), (6), and (7):

$$
r_{\text {crit }}=\frac{2 G m_{\mathrm{U}}}{(\Delta V)^{2}} 4 A \cos ^{2} \psi
$$

Although the orbits before and after the impact have the same velocity at $r_{\text {crit }}, a_{1}$ is slightly larger than $a_{2}$ [see Eq. (8)] due to the mass increment of the planet, but the energy of both orbits remains the same. From Eq. (7), the solutions for $r_{\text {crit }}$ are those with $\cos \psi<0$ since to keep the velocity modulus constant an impulse in the opposite direction to the instantaneous satellite motion is necessary. An impulse in the sense of the satellite motion would always lead to an increase in its instantaneous orbital velocity. When $\psi=180^{\circ}$, the upper bound of $r_{\text {crit }}$ is obtained. Then, $\vec{v}_{1}=-\vec{v}_{2}$. The lower bound is zero because it is not possible for $\vec{v}_{1}$ and $\vec{v}_{2}$ to have the same direction and sense in $r_{\text {crit }}$ unless the escape velocity and then $v_{1}$ and $v_{2}$ were infinite at this point.

From Eq. (10) and using Eq. (8), one obtains $a_{1}$ and $a_{2}$ for any value of $r$. The minimum eccentricity of the orbits before collision is $e_{1 \mathrm{~m}}=2(1-A)-1$ if $A \leq 0.5$, or $e_{1 \mathrm{~m}}=1-2(1-A)$ if $A \geq 0.5$, while the minimum eccentricity acquired by the orbits after collision is $e_{2 \mathrm{~m}}=2(1-B)-1$ if $B \leq 0.5$, or $e_{2 \mathrm{~m}}=$ $1-2(1-B)$ if $B \geq 0.5$. Through the upper and lower bounds of $r$, the upper and lower bounds of $a_{1}\left(a_{1 \mathrm{M}}\right.$ and $a_{1 \mathrm{~m}}$, respectively) and $a_{2}\left(a_{2 \mathrm{M}}\right.$ and $a_{2 \mathrm{~m}}$, respectively) may be determined as a function of $A$ and $B$ for given values of $m_{\mathrm{i}}, T_{0}$, and $\alpha$.

In the following, all the results are obtained taking $\alpha=70^{\circ}$ and $T_{0}=20 \mathrm{~h}$. These values of $\alpha$ and $T_{0}$ set the highest constraints on $a_{1 \mathrm{M}}$ and $a_{2 \mathrm{M}}$ and the lowest constraints on the orbital eccentricities. The smooth dependence of the results with the impactor mass (see, for example, Figs. 1-4) allows us to take $m_{\mathrm{i}}=1 m_{\oplus}$.

In Fig. 5, $a_{1 \mathrm{M}}$ is shown as a function of $A$ for $B=1, \alpha=70^{\circ}$, and $T_{0}=20 \mathrm{~h}$. Any satellite with orbital semiaxis greater than or equal to $a_{1 \mathrm{M}}$ is unbound at collision for any incident direction of the impactor and any other value of $\alpha$ and $T_{0}$ (inside the expected range of these parameters). If the five uranian satellites existed before collision at their present distances, they would have been unbound if their velocity was less than or equal to the circular velocity at impact $(A \leq 0.5)$. If their orbital velocity at impact

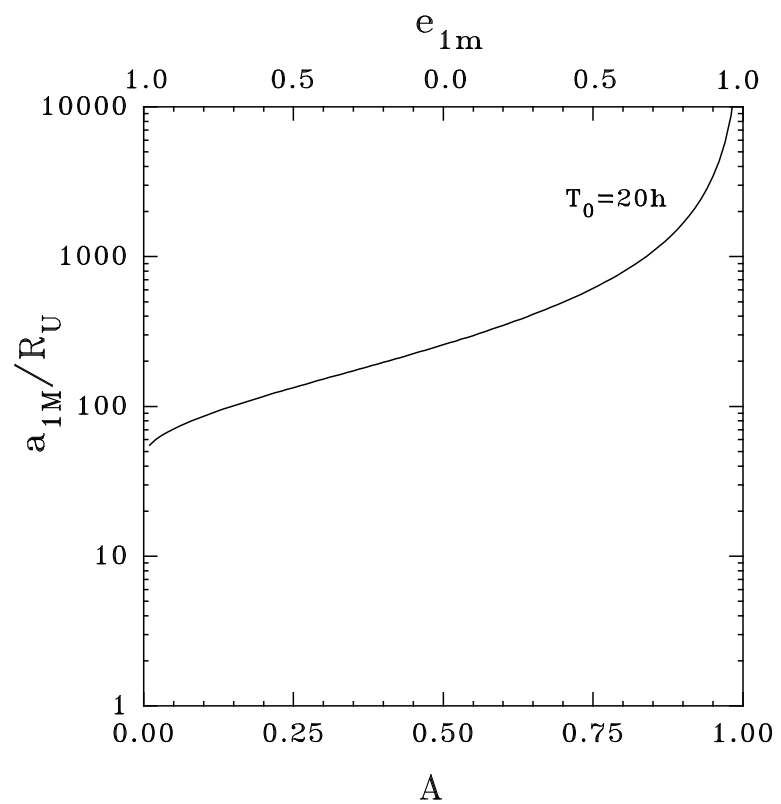

FIG. 5. Upper bound of the initial orbital semiaxis $\left(a_{1 \mathrm{M}}\right.$ in units of $R_{\mathrm{U}}=25,400 \mathrm{~km}$ ) from which any satellite in elliptical orbit is unbound as a function of $A$ for $m_{\mathrm{i}}=1 m_{\oplus}, T_{0}=20 \mathrm{~h}$, and $\alpha=70^{\circ} . A$ is the square of the ratio of the satellite's speed just before the impact to the escape velocity at the satellite's location just before the impact. $e_{1 \mathrm{~m}}$ is the minimum initial eccentricity of the orbits which are unbound at collision. 
had been larger $(A>0.5)$, they were close to pericenter and the satellites would have been transferred to new orbits, but probably the two outermost satellites would have been unbound.

If the outer uranian satellites existed before collision, they could have had inner or outer orbits transferred to their present distances at impact. All the preexisting satellites with different orbits and velocities received the same impulse at the moment of collision. We analyze the permitted transfers for each one of the known satellites independently, based on their current orbital properties.

For each $A$, we obtain the value of $B$ corresponding to the transfer to $a_{2 \mathrm{M}}=$ the actual semiaxis $a$ of S/1997 U1 (tabulated in Table I) for $\alpha=70^{\circ}$ and $T_{0}=20 \mathrm{~h}$. This value of $B$ provides the minimum eccentricity $e_{2 \mathrm{~m}}$ acquired by the orbit for any incident direction of the impactor (if $A<B^{\prime}$ ) or any incident direction of the impactor falling inside the permitted window (if $A>B^{\prime}$ ) and any $T_{0}$ and $\alpha$ (inside the expected range of these parameters). If this value of $e_{2 \mathrm{~m}}$ is less than or equal to the maximum eccentricity $e_{\max }$ of S/1997 U1 (tabulated in Table III), this satellite could have been transferred from the assumed initial condition " $A$ " to its present orbit. But if the resulting $e_{2 \mathrm{~m}}$ is greater than $e_{\max }$, the assumed transfer is not permitted and it could not have occurred. We carried out this procedure for every initial condition " $A$ " with each one of the known satellites. All the permitted transfers inside a range of $20 R_{\mathrm{U}}$ around each actual satellite's semiaxis ( $a_{2 \mathrm{M}}$ inside the range $a \pm 20 ; a$ taken from Table I) are displayed in Fig. 6.

The only permitted transfers for S/1999 U3 are those arising from the pericenter of a quasi-parabolic orbit $(0.9<A<1)$ or from a parabolic orbit $(A=1)$. Also, the minimum final eccentricity $e_{2 \mathrm{~m}}$ for the transfers from a quasi-parabolic orbit is equal to $e_{\max }(0.54)$. Then, this satellite would refute the hypothesis of its existence before the impact in an orbit bound to the planet. If we relate the existence of all the outer uranian moons to a common origin, we may conclude that the outer uranian system could not exist before the great collision. We will analyze this result in Section 6.

We now turn to the question of whether the giant collision itself could have provided a capture mechanism. Since all the permitted transfers with $A>B^{\prime}$ lead to a more bound orbit, this process might transform a temporary capture into a permanent one. Moreover, a permanent capture could even occur from a heliocentric orbit. All the satellites have permitted transfers with $A>B^{\prime}$ (see Fig. 6); one wonders if it could be possible to selfconsistently produce the permanent capture of all the moons in a single event.

For $A \geq 1$ the initial orbits are heliocentric. The transfers with $A$ close to 1 in Fig. 6 occur for values of $r \sim 200-350 R_{\mathrm{U}}$ for all the satellites. It is interesting to estimate the number of objects $N$ in heliocentric orbits at the time of the giant collision, at distances from Uranus less than or equal to $300 R_{\mathrm{U}}$. Assume that the giant collision occurred when Uranus was almost fully formed, meaning that its feeding zone was already depleted of primordial planetesimals. We assume that the objects passing near Uranus at that time were mainly escapees from the Kuiper belt. Using the impact rate onto Uranus and the distributions of velocities and diameters given by Levison et al. (2000), and assuming that the mass in the transneptunian region at the end of Solar System formation was 10 times its present mass, a back-ofthe-envelope calculation gives one object of diameter $D \geq 20 \mathrm{~km}$ passing at a distance $R \leq 300 R_{\mathrm{U}}$ from Uranus every $\sim 6$ years. This low rate of incoming objects makes the possibility of the capture of all the satellites from heliocentric orbits implausible. Even the capture of a single satellite (S/1999 U3) turns out to be difficult.

Temporary capture can lengthen the time which a passing body can spend near the planet (e.g., Vieira Neto and Winter 2001). Since S/1999 U3 could not have an orbit bound to the planet before collision, only the other four satellites could have been orbiting temporarily around the planet and transferred to permanent orbits by the collision. However, the existence of a forbidden region implies that all the satellites should have been in the same range of phase at impact ( $\psi$ must be in the range $100^{\circ}-260^{\circ}$ ).

In the context of the great collision scenario as a mechanism to produce the outer uranian system, a more plausible situation arises if we assume that the collision could produce the permanent capture of one (or two) parent object(s) which were orbiting temporarily around Uranus and that the present moons are the result of a collisional breakup occurring after the giant collision. This scenario will be analyzed in Section 6.

It should be noted that the collision itself would also provide a mechanism to transform a regular satellite into an irregular one. Although some transfers with $A=0.5$ are permitted (see Fig. 6), the initial semiaxes for these transfers fall outside $100 R_{\mathrm{U}}$ (much larger than the usual semiaxes of the regular satellites), making this possibility implausible.

\section{THE POSTCOLLISION ORBITAL EVOLUTION OF THE OUTER URANIAN MOONS DUE TO GAS DRAG}

In our previous calculations, we have assumed that the gravitational perturbations are the only effect capable of altering the orbital elements after the impact. However, dissipative mechanisms could change the orbital elements. If this were the case, the present orbital parameters of the outer uranian moons may not be primordial.

Three forms of dissipation of orbital energy are generally considered: tidal friction, gas drag, and collisions. Because of the large distance of the outer satellites from Uranus, the effect of tidal friction is negligible. Under the influence of gas drag the semimajor axis, eccentricity, and inclination of a satellite orbit all decrease. For a retrograde object, the inclination decreases in an algebraic sense, with the retrograde orbital plane changing toward $90^{\circ}$.

It is usually thought that the solar nebula dissipated before the end of the formation of Uranus and Neptune, since these planets 


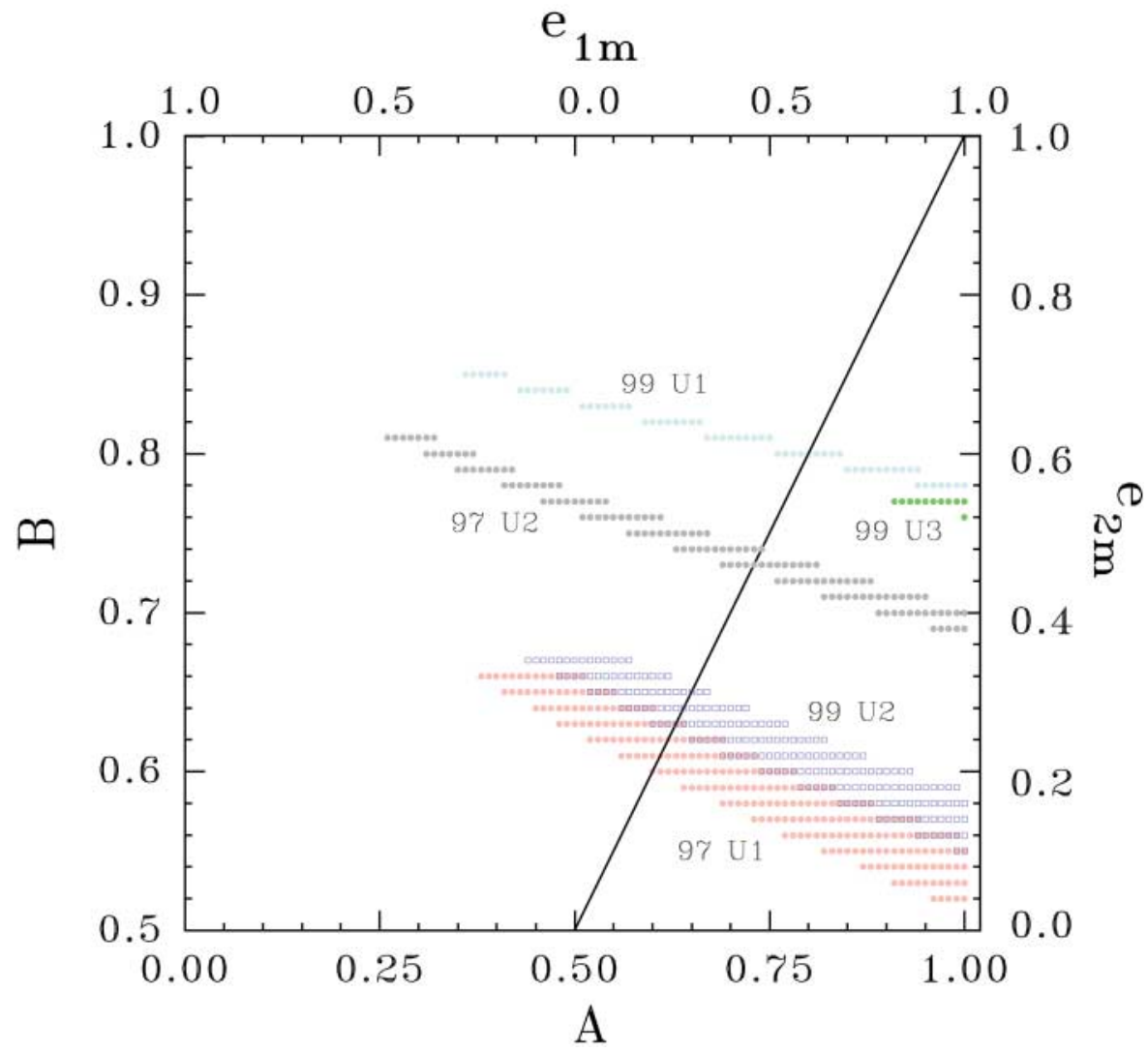

FIG. 6. The permitted transfers capable of producing the actual orbits of the outer uranian satellites for any incident direction of the impactor (if $A<B^{\prime}$ ) or any incident direction of the impactor inside the permitted window (if $A>B^{\prime}$ ) shown for $m_{\mathrm{i}}=1 m_{\oplus}, T_{0}=20 \mathrm{~h}$, and $\alpha=70^{\circ}$. $B$ is the square of the ratio of the satellite's speed just after the impact to the escape velocity at the satellite's location just after the impact. $e_{1 \mathrm{~m}}\left(e_{2 \mathrm{~m}}\right)$ is the minimum eccentricity of the orbits before (after) collision. The curve $A=B$ divides the upper region (the current orbits arise from inner orbits) and the lower region (the current orbits arise from outer orbits). (Turquoise) S/1999 U1; (green) S/1999 U3; (gray) S/1997 U2; (blue) S/1999 U2; (red) S/1997 U1. The only permitted transfers for S/1999 U3 are those arising from the pericenter of a quasi-parabolic orbit producing an orbital excentricity equal to its current maximum value. Note the narrow overlap of S/1999 U2 and S/1997 U1. There are no permitted transfers below $B=0.5$ for any of the satellites.

could not reach the runaway gas accretion phase (Bodenheimer and Pollack 1986, Pollack et al. 1996). The nebula was thus probably absent before the great collision occurred and the outer uranian satellites could not experience gas drag due to the solar nebula after the impact. However, within the core instability scenario, when the core mass has grown enough, a gaseous envelope of Uranus begins to form around the core, and the core and envelope continue to grow with the envelope extended to the accretion radius, which is $\sim 500 R_{\mathrm{U}}$ at the end of Uranus' formation. A satellite orbiting Uranus could suffer the effect of gas drag due to the planet's gas envelope. After termination of accretion, the envelope radius remains almost constant during a time scale of $10^{4}$ years and then contracts rapidly to $\sim 8 R_{\mathrm{U}}$ radii in $T=10^{5}$ years. The final contraction to the present-day planetary radius is on a slower time scale of $10^{8}$ years (Bodenheimer and Pollack 1986, Korycansky et al. 1990).

We will estimate the effect of gas drag suffered by outer uranian satellites since the end of the accretion process (after the impact) due to the presence of Uranus' extended envelope before its contraction to the present state. We are mainly interested in the possible damping of the orbital eccentricities and semiaxes of the known satellites.

A disk (where regular satellites might be later formed) could have formed out of a small amount of the outer envelope placed in orbit by the collision. The possible interaction between this disk and the outer satellites is beyond the scope of the present paper.

Following the procedure of Adachi et al. (1976), we obtain the time variations of the eccentricity $e$ and the semiaxis $a$ of each outer uranian satellite. The drag force per unit mass is expressed in the form

$$
F=-C \rho_{\mathrm{g}} v_{\mathrm{rel}}^{2}, \quad C=\frac{C_{\mathrm{D}} \pi r_{\mathrm{s}}^{2}}{2 m}
$$

where $v_{\text {rel }}$ is the relative velocity of the satellite with respect to the gas and $\rho_{\mathrm{g}}$ is the gas density. In computing the satellite mass $m$, a satellite density $\rho_{\mathrm{s}}$ of $1 \mathrm{~g} \mathrm{~cm}^{-3}$, characteristic of Centaurs and Kuiper belt objects (Gladman et al. 1998), is considered. The drag coefficient $C_{\mathrm{D}}$ is $\sim 1$ and $r_{\mathrm{s}}$ is the satellite radius (taken from Table I). 
To set upper bounds to the damping effect, we will maximize $v_{\text {rel }}$. As the actual rotation of Uranus is retrograde with respect to its orbital motion, the motion of retrograde outer uranian satellites is in the sense of Uranus' rotation. In this case, the assumption of a null gas velocity with respect to Uranus' center of mass will maximize the relative velocity. Thus, we will take $v_{\text {rel }}$ as the satellite orbital velocity. There is no force perpendicular to the orbital plane.

Assuming that the orbital elements are constant within the Keplerian period (the variations of $a$ and $e$ are very small), we consider the rates of change of the elements averaged over one period, that is,

$$
\begin{aligned}
\left\langle\frac{d a}{d t}\right\rangle= & -\frac{C}{\pi}\left(G\left(m_{\mathrm{i}}+m_{\mathrm{U}}\right) a\right)^{\frac{1}{2}} \int_{0}^{2 \pi} \frac{\rho_{\mathrm{g}}\left(e^{2}+1+2 e \cos \theta\right)^{\frac{3}{2}}}{(1+e \cos \theta)^{2}} d \theta \\
\left\langle\frac{d e}{d t}\right\rangle= & -\frac{C}{\pi}\left(1-e^{2}\right)\left(\frac{G\left(m_{\mathrm{i}}+m_{\mathrm{U}}\right)}{a}\right)^{\frac{1}{2}} \\
& \times \int_{0}^{2 \pi} \frac{\rho_{\mathrm{g}}(e+\cos \theta)\left(e^{2}+1+2 e \cos \theta\right)^{\frac{1}{2}}}{(1+e \cos \theta)^{2}} d \theta
\end{aligned}
$$

After the impact with the core, the envelope reacts hydrodynamically and a shock wave propagates away from the core, accelerating envelope gas outward. Korycansky et al. (1990) found that there was a sharp transition between the case where almost all the envelope mass remained and that where it was almost entirely dispersed. Their result implies that the impact would have had to have been one which did not disperse the envelope, as there would have been no nebular gas left to reaccrete. Behind the shock the gas falls back to the core, as it is not sufficiently heated to escape and the expected result is a gradual readjustment rather than a catastrophic transformation (Korycansky et al. 1990). Within this scenario, we assume that the envelope density profile after the impact quickly reaches the one it had before the impact. Thus, we fit, from Fig. 1 of Korycansky et al. (1990), the density profile of Uranus' envelope that corresponds to Uranus before the impact, $\rho_{\mathrm{g}}=10^{36} R^{-4}$ $\mathrm{g} \mathrm{cm}^{-3}$, with $R=a\left(1-e^{2}\right) /(1+e \cos \theta)$, with $a$ being measured in centimeters. For reference, at $R=100 R_{\mathrm{U}}, \rho_{\mathrm{g}}=2.4 \times$ $10^{-10} \mathrm{~g} \mathrm{~cm}^{-3}$. Since after termination of accretion the gas in the outer regions of the envelope contracts rapidly, Eqs. (13) have been integrated using the density distribution $\rho_{\mathrm{g}}$ over $10^{4}$ years.

Due to the uncertainties in the orbital elements, we have explored a range of values around the mean eccentricities given in Table III $\left(e=e_{\text {mean }} \pm 0.04\right)$ and around the actual semiaxes given in Table I ( $a=a \pm 20 R_{\mathrm{U}}$ ). The maximum damping of the orbital elements inside these ranges are tabulated in Table IV. The effect of gas drag on the satellites orbital evolution proves negligible. It should be noted that these variations are maximized, since a null gas velocity was assumed. If the envelope rotation were taken into account, the variation of these orbital elements would be even smaller. Therefore, we may conclude that the orbital elements of S/1997 U1, S/1997 U2, S/1999 U1, S/1999 U2, and S/1999 U3 may be considered as primordial.
TABLE IV

Orbital Damping due to Gas Drag

\begin{tabular}{lcc}
\hline Satellite & $d a / a$ & $d e / e$ \\
\hline S/1997 U1 & $1.9 \times 10^{-2}$ & $4.1 \times 10^{-2}$ \\
S/1997 U2 & $2.1 \times 10^{-2}$ & $1.8 \times 10^{-3}$ \\
S/1999 U1 & $5.7 \times 10^{-3}$ & $3.5 \times 10^{-3}$ \\
S/1999 U2 & $2.2 \times 10^{-2}$ & $4.2 \times 10^{-2}$ \\
S/1999 U3 & $1.5 \times 10^{-3}$ & $2.5 \times 10^{-3}$
\end{tabular}

To generalize these results, we also carried out the integrations for arbitrary initial conditions to see how gas drag could affect the orbits of any satellite orbiting Uranus at that time. For satellites with initial semiaxes between 300 and $380 R_{\mathrm{U}}$ and initial eccentricities $>0.7$, the pericenter crosses a region of very large density and the final state is usually a collision onto the planet. However, if the initial eccentricity is $<0.5$, the variation of the eccentricity is small $\left(<10^{-2}\right)$ and the semiaxes diminish only a few planetary radii. The transition between both regimes is very narrow, because of the deep profile of Uranus' atmospheric density. If the satellites were even farther from the planet (380-500 $R_{\mathrm{U}}$ ), they collide with the planet when the initial eccentricity is $>0.8-0.9$. For initial eccentricities $<0.7$, the variation of the eccentricity is $<10^{-2}$ and the semiaxes are again reduced only a small number of planetary radii.

\section{CONCLUSIONS}

The actual orbital properties of S/1999 U3 imply that this satellite could not exist before the impact (if it occurred at the end of Uranus' formation) in an orbit bound to the planet. Relating the origin of the outer uranian system to a common formation process, we conclude that the existence of these satellites implies either that their origin must be connected to one of the following scenarios (in order of plausibility) or that the giant collision did not occur:

1. The outer uranian moons had to be captured after collision. In this case, the problem is the nature of the dissipative forces able to transform a temporary capture into a permanent one, since the impact is assumed to have occurred very late in the stage of the planet accretion process, or still later, when a dense nebula to allow the permanent capture due to gas drag seems to be inplausible and there is no more mass to be accreted by the planet to enhance its Hill sphere of action. A collisional scenario after the giant impact could, in principle, account for the capture and breakup of the outer uranian moons. Recent photometric and spectroscopic studies have revealed important clues: a significant variability in the lightcurve of S/1997 U1 (Maris et al. 2001) and the peculiar spectrum of S/1997 U2 (Romon et al. 2001) could suggest an irregular surface for these satellites, which favors a collisional scenario. Probably, S/1997 U1 and S/1999 U2 belong to a parent body, since their orbits are almost the same. The similar orbital elements of S/1999 U1 
and S/1999 U3 also suggest that both satellites may belong to a parent object.

Uranus appears to have at present three or two families (three or two parent objects) of retrograde outer satellites. The fact that the five moons have similar orbital inclinations could suggest a single parent body. The largest regions of the initial-conditions space with the longest temporary capture times for retrograde uranian satellites occur at an inclination of $160^{\circ}$ (Vieira Neto and Winter 2001). The current orbital inclinations of the known uranian moons are close to this value (although somewhat less, suggesting the action of some dissipative process). This indicates that the transition to their actual state is probable for each parent body (and even for each satellite) independently.

2. These satellites are remnants of larger satellites which could have been captured before collision. In this case, one, two, or three parent objects could have been captured by any mechanism before collision and transferred to new orbits at impact. But the breakup process had to occur after the great collision.

3. The giant collision itself produced the capture of one (or two) parent objects and the known moons are the result of a breakup process occurring after the large impact. However, the fact that the longest temporary capture times for retrograde uranian satellites occur for inclinations near the actual satellites' inclinations (Vieira Neto and Winter 2001) suggests that the capture would be due to some dissipative process after the giant impact instead of this disruptive mechanism.

4. The existence of the outer uranian system before collision could be possible assuming that the giant collision occurred after the accretion process and later in the evolution of the planet when it had almost reached its present state. In this case, the impact parameter $b$ of the collision could be much larger than that considered in this paper, allowing permitted transfers for S/1999 U3 from an orbit bound to the planet. This scenario is less plausible than the others already cited because large impacts are usually believed to occur at the end of the accretion process and not much later.

5. The outer uranian system could have existed before the giant collision at the end of Uranus' formation if $T_{0} \sim T_{0 \mathrm{~m}}=45 \mathrm{~h}$ and the spin axis inclination due to the impact $\alpha$ is not larger than $70^{\circ}$. The permitted transfers of Fig. 6 would increase if we take the parameters $\alpha=70^{\circ}$ and $T_{0}=T_{0 \mathrm{~m}}$. Strong constraints on the initial status of rotation of Uranus are set in this case. Some of the present spin axis inclination of the planet must be due to another process. Although the permitted transfers for S/1999 U3 remain very restrictive even in this case, initial values of $A \geq 0.65$ would be able to produce a final orbit like Prospero's. There are permitted transfers for S/1999 U3 from an orbit bound to the planet only if $T_{0}=T_{0 \mathrm{~m}}$. This restriction in the initial period makes this scenario the most implausible.

Cases 4 and 5 are the less plausible, but they allow more permitted transfers for all the satellites. Some of them arise from very small initial semiaxes characteristic of regular satellites. Other transfers could occur from outer orbits with very large values of $r$ where the number of objects coming from the Kuiper belt would then be larger (i.e., if $r$ is $2000 R_{\mathrm{U}}, N \sim 14$ objects per year at the end of the accretion process for case 5), increasing the probability of capture of objects in heliocentric orbits via the giant collision.

The orbital properties of S/1999 U3 set such strong constraints on the great collision scenario that we also have to consider the possibility that a giant collision did not occur. Then, other mechanisms able to produce the large spin axis inclination of the planet should be investigated.

\section{ACKNOWLEDGMENTS}

We appreciate the useful suggestions by B. Gladman and A. Dobrovolskis, who as referees have helped us to greatly improve this paper. We thank R. A. Jacobson for providing his results and also for useful comments and P. D. Nicholson and J. A. Burns for helpful information. M. G. Parisi thanks O. G. Benvenuto for his collaboration.

\section{REFERENCES}

Adachi, I., C. Hayashi, and K. Nakazawa 1976. The gas drag effect on the elliptic motion of a solid body in the primordial solar nebulae. Prog. Theo. Phys. 56, 1756-1771.

Bodenheimer, P., and J. B. Pollack 1986. Calculations of the accretion and evolution of giant planets: The effect of solid cores. Icarus 67, 391-408.

Brunini, A. 1993. Orbital evolution of the terrestrial planets as a result of close encounters and collisions with planet-crossing asteroids. Planet. Space Sci. 41, 747-751.

Brunini, A. 1995a. A possible constraint to Uranus' great collision. Planet. Space Sci. 43, 1019-1021.

Brunini, A. 1995b. Capture of planetesimals by the giant planets. Earth, Moon, Planets 71, 281-284.

Brunini, A. 1996. On the satellite capture problem. Celest. Mech. Dynam. Astron. 64, 79-92.

Brunini, A., and J. A. Fernandez 1999. Numerical simulations of the accretion of Uranus and Neptune. Planet. Space Sci. 47, 591-605.

Byl, J., and M. W. Ovenden 1975. On the satellite capture problem. Mon. Not. R. Astron. Soc. 173, 579-584.

Catullo, V., V. Zappala, P. Farinella, and P. Paolicchi 1984. Analysis of the shape distribution of asteroids. Astron. Astrophys. 138, 464-468.

Colombo, G., and F. A. Franklin 1971. On the formation of the outer satellite groups of Jupiter. Icarus 15, 186-189.

Degewij, J., L. E. Andersson, and B. Zellner 1980. Photometric properties of outer planetary satellites. Icarus 44, 520-540.

Dones, L., and S. Tremaine 1993. On the origin of planetary spins. Icarus $\mathbf{1 0 3 ,}$ 67-92.

Duncan, M. J., and H. F. Levison 1997. A scattered comet disk and the origin of Jupiter family comets. Science 276, 1670-1674.

Gil Hutton, R. 2002. Color diversity among Kuiper belt objects: The collisional resurfacing model revisited. Planet. Space Sci. 50, 57-62.

Gladman, B. J., P. D. Nicholson, J. A. Burns, and J. J. Kavelaars 1997. Satellites of Uranus. IAU Circ. 6764.

Gladman, B. J., P. D. Nicholson, J. A. Burns, J. J. Kavelaars, B. J. Marsden, G. V. Williams, and W. B. Offutt 1998. Discovery of two distant irregular moons of Uranus. Nature 392, 897-899.

Gladman, B. J., P. D. Nicholson, J. A. Burns, and J. J. Kavelaars 1999. Probable new satellites of Uranus. IAU Circ. 7248. 
Gladman, B. J., J. J. Kavelaars, M. Holman, J.-M. Petit, H. Scholl, P. D. Nicholson, and J. A. Burns 2000. The Discovery of Uranus XIX, XX, and XXI. Icarus. 147, 320-324; erratum 148, 320 (2000).

Gladman, B. J., J. J. Kavelaars, M. Holman, P. D. Nicholson, J. A. Burns, C. W. Hergenrother, J.-M. Petit, B. J. Marsden, R. Jacobson, W. Gray, and T. Grav 2001. Discovery of 12 satellites of Saturn exhibiting orbital clustering. Nature 412, 163-166.

Goldreich, P., N. Murray, P. Y. Longaretti, and D. Bandfield 1989. Neptune's story. Science 245, 500-504.

Green, D. 2001. Satellites of Jupiter. IAU Circ. 7555.

Greenberg, R. 1974. Outcomes of tidal evolution for orbits with arbitrary inclination. Icarus 23, 51-58.

Harris, A. W., and W. R. Ward 1982. Dynamical constraints on the formation and evolution of planetary bodies. Annu. Rev. Earth Planet. Sci. 10, 61-108.

Heppenheimer, T. A. 1975. On the presumed capture origin of Jupiter's outer satellites. Icarus 24, 172-180.

Heppenheimer, T. A., and C. Porco 1977. New contributions to the problem of capture. Icarus 30, 385-401.

Huang, T. Y., and K. A. Innanen 1983. The gravitational escape/capture of planetary satellites. Astron. J. 88, 1537-1548.

Jacobson, R. A. 1999. The determination and long term integration of the orbits of Caliban and Sycorax. Bull. Am. Astron. Soc. 31, 1224.

Jacobson, R. A. 2001. URA062. JPL satellite ephemeris.

Kavelaars, J. J., B. J. Gladman, M. Holman, J.-M. Petit, and H. Scholl 1999. Probable new satellites of Uranus. IAU Circ. 7230.

Korycansky, D. G., P. Bodenheimer, P. Cassen, and J. B. Pollack 1990. Onedimensional calculations of a large impact on Uranus. Icarus 84, 528-541.

Kubo-Oka, T., and K. Nakazawa 1995. Gradual increase in the obliquity of Uranus due to tidal interaction with a hypothetical retrograde satellite. Icarus 114, 21-32.

Laskar, J., and P. Robulet 1993. The chaotic obliquity of the planets, Nature 361, $608-612$

Levison, H. F., J. J. Lissauer, and M. J. Duncan 1998. Modeling the diversity of outer planetary systems. Astron. J. 116, 1998-2014.

Levison, H. F., M. J. Duncan, K. Zahnle, M. Holman, and L. Dones 2000. Planetary impact rates from ecliptic comets. Icarus 143, 415-420.

Lissauer, J. J. 1993. Planet formation. Annu. Rev. Astron. Astrophys. 31, 129174

Lissauer, J. J., and D. M. Kary 1991. The origin of the systematic component of planetary rotation. Icarus $\mathbf{9 4}, 126-159$.

Lissauer, J. J., and V. Safronov 1991. The random component of planetary rotation. Icarus $\mathbf{9 3}, 288-297$.

Lissauer, J. J., A. F. Berman, Y. Greenzweig, and D. M. Kary 1997. Accretion of mass and spin angular momentum by a planet on an eccentric orbit. Icarus 127, 65-92.

Luu, J. 1991. CCD photometry and spectroscopy of the outer jovian satellites. Astron. J. 102, 1213-1225.

Luu, J. 1997. Twice in a blue moon. Nature 390, 441-443.

Luu, J., and D. Jewitt 1996. Color diversity among the Centaurs and Kuiper belt objects. Astron. J. 112, 2310-2318.

Maris, M., G. Carraro, G. Cremonese, and M. Fulle 2001. Multicolor photometry of the Uranus irregular satellites Sycorax and Caliban. Astron. J. 121, 28002803.

Marsden, B. G. 2000a. S/1999 U1. IAU Circ. 7450.

Marsden, B. G. 2000b. S/1999 U2. IAU Circ. 7473.

Marsden, B. G. 2000c. S/1999 U3. IAU Circ. 7447.

Marsden, B. G. 2000d. S/1999 J1. IAU Circ. 7460.
Marsden, B. G. 2000e. S/2000 J1 = S/1975 J1. IAU Circ. 7525.

Marsden, B. G., and G. V. Williams 1997. Satellites of Uranus. IAU Circ. 6765.

Marsden, B. G., G. V. Williams, and K. Aksnes 1998a. S/1997 U2. IAU Circ. 6869.

Marsden, B. G., G. V. Williams, and K. Aksnes 1998b. S/1997 U1. IAU Circ. 6870 .

McKinnon, W. B., and A. C. Leith 1995. Gas drag and the orbital evolution of a captured Triton. Icarus 118, 392-413.

Morbidelli, A. 1997. Chaotic diffusion and the origin of comets from the $2 / 3$ resonance in the Kuiper belt. Icarus 127, 1-12.

Parisi, M. G., and A. Brunini 1996a. Dynamical consequences of Uranus' great collision. In Chaos in Gravitational N-Body Systems (J. C. Muzzio, S. FerrazMello, and J. Henrard, Eds.), pp. 291-296. Kluwer Academic, Dordrecht.

Parisi, M. G., and A. Brunini 1996b. The mass of large impactors. In From Stardust to Planetesimals: Contributed Papers (M. E. Kress, A. G. G. M. Tielens, and Y. Pendleton, Eds.), NASA Conference publications No. 3343, pp. 201-204. Natl. Aeronautics \& Space Admin., Washington, DC.

Parisi, M. G., and A. Brunini 1997. Constraints to Uranus' great collision II. Planet. Space Sci. 45, 181-187.

Parisi, M. G., and A. Brunini 1999. Dynamical constraints to the masses of large planetesimals. Planet. Space Sci. 47, 607-617.

Pollack, J. B., J. A. Burns, and M. E. Tauber 1979. Gas drag in primordial circumplanetary envelopes: A mechanism for satellite capture. Icarus 37, 587-611.

Pollack, J. B., J. I. Lunine, and W. C. Tittemore 1991. Origin of the uranian satellites. In Uranus (J. T. Bergtralh, E. D. Miner, and M. S. Mattews, Eds.), pp. 469-512. Univ. of Arizona Press, Tucson.

Pollack, J. B., O. Hubickyj, P. Bodenheimer, J. J. Lissauer, M. Podolak, and Y. Greenzweig 1996. Formation of the giant planets by concurrent accretion of solid and gas. Icarus 124, 62-85.

Romon, J., C. de Bergh, M. A. Barucci, A. Doressoundiram, J.-G. Cuby, A. Le Bras, S. Douté, and B. Schmitt 2001. Photometric and spectroscopic observations of Sycorax, satellite of Uranus. Astron. Astrophys. 376, 310 315 .

Saha, P., and S. Tremaine 1993. The orbits of the retrograde jovian satellites. Icarus 106, 549-562.

Safronov, V. S. 1969. Evolution of the Protoplanetary Cloud and Formation of The Earth and the Planets. NASA TTF-677. Natl. Aeronautics \& Space Admin., Washington, DC.

Slattery, W. L., W. Benz, and A. G. W. Cameron 1992. Giant impacts on a primitive Uranus. Icarus 99, 167-174.

Stevenson, D. J., A. W. Harris, and J. I. Lunine 1986. Origins of satellites. In Satellites (J. A. Burns and M. S. Matthews, Eds.), pp. 39-88. Univ. of Arizona Press, Tucson.

Tholen, D. J., and B. Zellner 1984. Multicolor photometry of outer jovian satellites. Icarus 58, 246-253.

Tremaine, S. 1991. On the origin of the obliquities of the outer planets. Icarus 89, 85-92.

Tsui, K. H. 1999. Satellite capture by scattering of an existing massive planetary satellite. Planet. Space Sci. 47, 917-920.

Vieira Neto, E., and O. C. Winter 2001. Time analysis for temporary gravitational capture: Satellites of Uranus. Astron. J. 122, 440-448.

Wilson, P. D. 1997. Models of Organic-rich Surfaces in the Outer Solar System. Thesis, Cornell University, Ithaca, New York.

Ziglina, I. N. 1976. Effect on eccentricity of a planet's orbit of its encounters with bodies of the swarm. Sov. Astron. 20, 730-733.

Ziglina, I. N., and V. S. Safronov 1976. Averaging of the orbital eccentricities of bodies which are accumulating into a planet. Sov. Astron. 20, 244-248. 